Research Paper

\title{
Combined metformin and insulin treatment reverses metabolically impaired omental adipogenesis and accumulation of 4-hydroxynonenal in obese diabetic patients
}

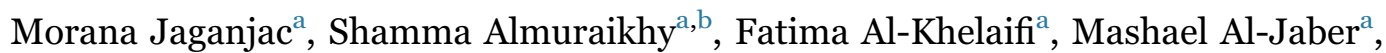 \\ Moataz Bashah $^{\mathrm{c}}$, Nayef A. Mazloum ${ }^{\mathrm{d}}$, Kamelija Zarkovic ${ }^{\mathrm{e}}$, Neven Zarkovic ${ }^{\mathrm{f}}$, Georg Waeg ${ }^{\mathrm{g}}$, \\ Wael Kafienah $^{\mathrm{b}, *}$, Mohamed A. Elrayess ${ }^{\mathrm{a}, *}$ \\ a Anti-Doping Lab Qatar, Sports City, Doha, Qatar \\ b School of Cellular and Molecular Medicine, University of Bristol, Bristol, United Kingdom \\ c Bariatric and Metabolic Surgery, Hamad Medical Corporation, Doha, Qatar \\ d Microbiology and Immunology, Weill Cornell Medicine-Qatar, Doha, Qatar \\ e Department of Pathology, Medical Faculty University of Zagreb, Clinical Hospital Centre Zagreb, Zagreb, Croatia \\ ${ }^{\mathrm{f}}$ Laboratory for Oxidative Stress, Rudjer Boskovic Institute, Zagreb, Croatia \\ ${ }^{\mathrm{g}}$ Karl Franzen's University of Graz, Institute of Molecular Biosciences, Austria
}

\section{A R T I C L E I N F O}

\section{Keywords:}

Obesity

Insulin resistance

T2DM

Omental fat

Adipogenesis

4-hydroxynonenal

Metformin

Insulin

\begin{abstract}
A B S T R A C T
Objective: Obesity-associated impaired fat accumulation in the visceral adipose tissue can lead to ectopic fat deposition and increased risk of insulin resistance and type 2 diabetes mellitus (T2DM). This study investigated whether impaired adipogenesis of omental (OM) adipose tissues and elevated 4-hydroxynonenal (4-HNE) accumulation contribute to this process, and if combined metformin and insulin treatment in T2DM patients could rescue this phenotype.

Methods: OM adipose tissues were obtained from forty clinically well characterized obese individuals during weight reduction surgery. Levels of 4-HNE protein adducts, adipocyte size and number of macrophages were determined within these tissues by immunohistochemistry. Adipogenic capacity and gene expression profiles were assessed in preadipocytes derived from these tissues in relation to insulin resistance and in response to 4HNE, metformin or combined metformin and insulin treatment.

Results: Preadipocytes isolated from insulin resistant (IR) and T2DM individuals exhibited lower adipogenesis, marked by upregulation of anti-adipogenic genes, compared to preadipocytes derived from insulin sensitive (IS) individuals. Impaired adipogenesis was also associated with increased 4-HNE levels, smaller adipocytes and greater macrophage presence in the adipose tissues. Within the T2DM group, preadipocytes from combined metformin and insulin treated subset showed better in vitro adipogenesis compared to metformin alone, which was associated with less presence of macrophages and 4-HNE in the adipose tissues. Treatment of preadipocytes in vitro with 4-HNE reduced their adipogenesis and increased proliferation, even in the presence of metformin, which was partially rescued by the presence of insulin.

Conclusion: This study reveals involvement of 4-HNE in the impaired OM adipogenesis-associated with insulin resistance and T2DM and provides a proof of concept that this impairment can be reversed by the synergistic action of insulin and metformin. Further studies are needed to evaluate involvement of 4-HNE in metabolically impaired abdominal adipogenesis and to confirm benefits of combined metformin-insulin therapy in T2DM patients.
\end{abstract}

\section{Introduction}

Obesity increases the risk of insulin resistance and type 2 diabetes mellitus (T2DM) [1]. However, some obese individuals, often referred to as the insulin sensitive (IS) or metabolically healthy obese (MHO), exhibit a lower risk of these diseases than predicted by their obesity [2]. Understanding the mechanisms underlying the protection found in IS obesity could help individuals suffering from pathological obesity.

\footnotetext{
* Corresponding authors.

E-mail addresses: W.Z.Kafienah@bristol.ac.uk (W. Kafienah), melrayess@adlqatar.qa (M.A. Elrayess).
} 
Obesity is characterized by increased size of adipose tissue through hypertrophy and hyperplasia of adipocytes [3]. Preadipocytes, an abundant cell population within the adipose tissue, replenish the adipocyte pool through adipogenesis [4]. Superior adipogenesis of preadipocytes isolated from sub-cutaneous (SC) adipose tissues taken from IS obese individuals compared to insulin resistant (IR) counterparts was recently suggested to play a role in the protection process of IS obesity, which is partially mediated by lower IL-6 secretion and oxidative stress $[5,6]$. Obesity-associated oxidative stress leads to elevated reactive oxygen species (ROS) production causing lipid peroxidation within the adipose tissue [7] and accumulation of reactive aldehydes [8,9]. Elevated 4-hydroxynonenal (4-HNE), a bioactive lipid peroxidation product, leads to progressive impairment of cell structure and function via formation of stable 4-hydroxyalkenals with proteins, phospholipids and DNA [10,11]. Elevation of 4-HNE has been associated with impaired adipogenesis, insulin resistance, atherosclerosis and even obesity of apparently healthy people [6,12-15].

Metformin (dimethylbiguanidine), the most widely used drug for the treatment of T2DM $[16,17]$, is an insulin-sensitizing agent that provides glycemic control, especially in obese individuals [18]. Metformin can reduce adipose tissue size in vivo [19] and in vitro by inhibiting adipogenesis, decreasing lipogenic gene expression and increasing AMPK activity and glucose intake [20-22]. Metformin is frequently given to T2DM in combination with insulin [23]. Previous studies have shown that intensive insulin therapy reverses the decrease in adipocyte glucose transport activity in T2DM [24] and counters inflammation by decreasing levels of IL-6 and TNFa [25]. Insulin is commonly used in in vitro studies to induce adipogenesis, as it triggers a series of transcription factors that drive differentiation of preadipocytes into mature adipocytes including phosphorylation and activation of CREB [26] and PI 3-kinase [27].

Characterization of adipogenesis in the omental (OM) adipose tissues, a depot that is associated with increased metabolic disease risk [28], in IS, IR and T2DM obese individuals remains to be elucidated. Additionally, the effects of metformin on human OM adipogenesis has not been tested, although metformin-mediated glucose uptake by SC and OM adipocytes was previously described [29]. Comparing adipogenesis of OM-derived preadipocytes between IS and IR individuals and the impact of local 4-HNE on this process in primary cultures would provide valuable insights into the potentially protective mechanism associated with the IS group. The aims of the study were to test three hypotheses: 1) adipogenic capacity of OM preadipocyte is impaired in IR and T2DM individuals compared to IS subjects, 2) this impairment is accompanied by elevated 4-HNE accumulation in the adipose tissue that can directly inhibit adipogenesis, and 3) a combination of metformin/insulin treatment can rescue impaired OM adipogenesis.

\section{Materials and methods}

\subsection{Materials}

IL-6 and leptin ELISAs were purchased from R\&D systems (Abingdon, UK) and Insulin ELISA from Mercodia Diagnostics (Sweden). Diaminobenzidine tetrahydrochloride (DAPI) and LipidTOX $^{\mathrm{TM}}$ Green Neutral Lipid were obtained from Life Technologies (Warrington, UK). RT2 Profiler human adipogenesis PCR arrays and cDNA synthesis kits were purchased from SABiosciences-Qiagen, (Hilden, Germany). Other chemicals and reagents were purchased from Sigma (Germany) unless otherwise indicated.

\subsection{Cohort}

Patient recruitment criteria were previously published [5]. Briefly, forty consented obese patients (20 females and 20 males, matched for age and BMI) undergoing weight reduction surgery at Hamad Medical Corporation (HMC) (Doha, Qatar) were recruited. Protocols were approved by Institutional Review Boards of HMC and ADLQ (SCHADL-070, SCH-JOINT-111). Blood was collected prior to operation and $1-5 \mathrm{~g}$ OM adipose tissues biopsies were collected during the surgery. Plasma cholesterol, fasting glucose, Hba1c and liver function enzymes were measured by COBAS INTEGRA (Roche Diagnostics, Basil). IL-6, leptin and insulin were determined using commercially available ELISA. Insulin resistance was computed by homeostatic model assessment (HOMA-IR) [30] using 30th percentile (HOMA$\mathrm{IR}=2.4$ ) as a threshold point. Accordingly, subjects were dichotomized into IS (HOMA-IR $<2.4, \mathrm{n}=8$ ) and IR (HOMA-IR $>2.4, \mathrm{n}=32$ ), including 11 subjects with T2DM ( 5 on metformin only, 5 on metformin and insulin injection and 1 diet treated).

\subsection{Preadipocytes culture and differentiation}

Stromal vascular fraction (SVF) cells were obtained by collagenase digestion of OM adipose tissues as described previously [31]. Cell pellets were re-suspended in stromal medium containing DMEM-F12 supplemented with 10\% FBS and Penicillin/Streptomycin, then maintained at $37{ }^{\circ} \mathrm{C}$ with $5 \% \mathrm{CO}_{2}$ until confluence. To induce differentiation, early passaged SVF-derived preadipocytes (passages $1-3$ ) were grown at $2 \times 10^{4} / \mathrm{cm}^{2}$ in stromal medium overnight then incubated in differentiation medium (DMEM-F12, 3\% FBS, $33 \mu \mathrm{M}$ biotin, $17 \mu \mathrm{M}$ Dpantothenate, $1 \mu \mathrm{M}$ dexamethasone, $250 \mu \mathrm{M}$ of methylisobutylxanthine, $0.1 \mu \mathrm{M}$ human insulin, $5 \mu \mathrm{M}$ of PPAR $\gamma$ agonist, rosiglitazone) for 7 days, followed by 12 days in maintenance medium containing same components as differentiation medium omitting methylisobutylxanthine and rosiglitazone [32]. Differentiation potential (adipogenic capacity) was determined as a percentage of lipidtox positive stained cells to total number of stained nuclei (DAPI). For experiments investigating the effect of 4-HNE, metformin, insulin and their various combinations on differentiation, cells were grown as above in the absence or presence of repeated (every 3 days) of $10 \mu \mathrm{M} 4-\mathrm{HNE}, 1 \mathrm{mM}$ metformin, $0.1 \mu \mathrm{M}$ human insulin or their combinations for the entire differentiation and maintenance periods.

\subsection{Gene expression studies}

RNA was extracted from differentiated OM adipocytes using Trizol following manufacturer's instructions. One microgram of RNA was used to synthetize cDNA and gene expression profiling was determined using RT2 Profiler human adipogenesis PCR arrays by assessing mRNA levels of 84 genes, including five "housekeeping genes" according to manufacturer's protocol. The list of genes included Cyclin D1 (CCND1), Fatty Acid Binding Protein 4 (FABP4), TSC22 Domain Family Member 3 (TSC22D3) and Peroxisome Proliferator-Activated Receptor Gamma Coactivator 1 Beta (PPARGC1B, also known as PGC1beta). Data were normalized with the internal housekeeping genes and $\Delta \Delta \mathrm{Ct}$ was calculated using $\Delta \mathrm{Ct}$ from IS as the control group according to manufacturer's protocol.

\subsection{Measurement of ROS production}

Intracellular ROS levels were assessed using $2^{\prime}, 7^{\prime}$-dichlorofluorescein-diacetate (DCFH-DA, Fluka) probe as described previously [33]. Briefly, cells were incubated with DCFH-DA $(10 \mu \mathrm{M})$ in the HBSS for 30 min followed by removal of the probe and treatment with 4-HNE $(10 \mu \mathrm{M})$ in stromal medium containing $3 \%$ FBS. The fluorescence intensity (relative fluorescence units, RFU) was measured every hour for $12 \mathrm{~h}$ using TECAN Infinite M200 PRO plate reader equipped with gas control mode to maintain $37^{\circ} \mathrm{C}$ and $5 \% \mathrm{CO}_{2}$. 


\subsection{4-HNE staining}

Immunohistochemical analysis of adipose tissues biopsies was performed as previously described [6]. Briefly, formalin-fixed and paraffin-embedded sections made from paraffin blocks were stained with Haematoxylin/Eosin (HE) or with a monoclonal antibody specific for the HNE-histidine epitope in HNE-protein (peptide) conjugates. For the immunohistochemical detection of the HNE-protein adducts the immunoperoxidase technique was used using EnVision kit (Dako, Denmark) as described previously [34]. HNE positivity was estimated using a semi-quantitative method by an experienced pathologist $(-0 \%$ positive cells, $+<5 \%$ positive cells, $++5-25 \%$ positive cells, $+++25-$ $50 \%$ positive cells, $++++>50 \%$ positive cells). The presence of HNEprotein adducts in connective tissue, inflammation and in blood vessels was defined as negative (-) in the absence of the HNE-protein adducts, or as low positive $(+,++)$ or high positive $(+++,++++)$ in the presence of the HNE-protein adducts [35].

\subsection{Statistical analysis}

Comparisons were performed using t-test, Wilcoxon-MannWhitney and 1-way ANOVA in IBM SPSS statistics 21. Significance was defined as $\mathrm{P} \leq 0.05$. Power calculations indicated that the present sample size $(n=40)$ had $80 \%$ power to detect a minimal difference of $30 \%$ in mean differentiation capacity of IS versus IR+ type 2 diabetes mellitus with $35 \%$ deviation from mean value (sigma) at a level of $\alpha=0.05$.

\section{Results}

\subsection{Insulin resistance-associated impairment of OM adipogenesis}

Forty obese and morbidly obese (BMI $\left.=43.6 \pm 6.7 \mathrm{~kg} \mathrm{~m}^{-2}\right)$, young (age $=35.6 \pm 11.9$ years) patients were included in this study. General characteristics of the cohort were previously published [5] and shown for the studied group in Supplementary Table S1. Both metformin monotherapy or metformin and insulin combined therapy exhibited a comparable glycemic control over T2DM patients as indicated by the matching Hba1c levels, despite the non-significant elevation in FPG and insulin levels in the combined treatment group. The metformin and insulin treatment group showed significant reduction in systemic IL-6 levels compared to patients treated with metformin monotherapy (Table S1).

The adipogenic capacity of SVF-derived preadipocytes was assessed in all participants. Compared to IS individuals, IR and T2DM patients exhibited reduced OM adipogenic capacity by $26.3 \% \quad(\mathrm{p}<0.01)$ (Fig. 1A, B). As the obtained data revealed that the adipogenic capacity was equally reduced in IR and T2DM groups (Fig. 1B), these two groups were further combined when conducting gene expression comparison in order to increase the power (Fig. 1C). The reduced adipogenic capacity of IR \& T2DM-derived preadipocytes was associated with up-regulation of anti-adipogenic genes CCND1, FABP4 and TSC22D3 and down-regulation of the pro-adipogenic gene PPARGC1B (Fig. 1C). Interestingly, preadipocytes expanded from T2DM patients who were treated with a combination of metformin and insulin showed greater adipogenic capacity than those treated with metformin monotherapy (Fig. 1D), as well as the diet-treated individual (4\%). The reversing of the adipogenic capacity in the combined treatment group (20.3\%), compared to metformin monotherapy group (1.7\%) (Fig. 1D), is still lower than that of the IS group (43.1\%, Fig. 1B), perhaps due to the anti-adipogenic effect of metformin.

3.2. Insulin resistance-associated 4-HNE staining, adipocyte size and macrophages infiltration within $O M$ adipose tissues

A semi-quantitative immunohistochemical analysis of adipose tissues from randomly selected samples revealed that disease progression was associated with increased 4-HNE levels in adipocytes (Fig. 2A), smaller size of the adipocytes (Fig. 2B) and reduced macrophage infiltration (Fig. 2C). However, a lower 4-HNE-immunopositivity was detected in $\mathrm{OM}$ tissues from metformin and insulin treated T2DM patients when compared to those treated with metformin monotherapy (Fig. 2D). In the metformin monotherapy group, the 4-HNE immunopositivity was most pronounced in the cellular membranes of the adipocytes (brown color) and occasionally in inflammatory cells infiltrating the adipose tissue, as indicated by arrows (Fig. 2D top left image). Furthermore, high 4-HNE immunopositivity was also detected in the walls of blood vessels and in the perivascular interstitial connective tissue as indicated by arrows (Fig. 2D top right image). Only a weak immunopositivity for 4-HNE was seen in the metformin and insulin group in the membranes of some adipocytes and the blood vessel walls, while 4-HNE was mostly absent in their OM interstitial connective tissue (indicated by arrow) as visualized by the blue color of the hematoxylin contrast (Fig. 2D bottom left image). Furthermore, lower levels of 4-HNE immunopositivity were usually discontinuously present in the membranes of the adipocytes and the interstitial blood vessels in this group (indicated by arrow) (Fig. 2D bottom right image).

\subsection{Effect of 4-HNE and metformin treatment in vitro on ROS production and $O M$ adipogenesis in the presence of insulin}

Preadipocytes were treated with metformin, 4-HNE or their combinations in the presence or absence of insulin (Fig. 3A). Both 4HNE and metformin treatments caused significant elevation in ROS production in the absence or presence of insulin (Fig. 3B). Furthermore, 4-HNE, metformin given alone or combined with 4HNE caused reduced adipogenic capacity that was partially rescued by co-treatment with insulin (Fig. 3A, C). The lipidtox signal in cells treated with insulin and 4-HNE in the presence of metformin was lower than when metformin was omitted (Fig. 3A) as quantified in Fig. 3C. This is probably due to inhibition of adipogenic capacity in response to metformin, despite partial rescue due to presence of insulin. The reduced adipogenic capacity with 4-HNE treatment had no effect on cell size, whilst metformin treatment decreased the size of differentiated cells significantly (Fig. 3D). Interestingly, 4-HNE treatment caused a significant increase in cell number $(\mathrm{p}<0.05)$, a trend that was also seen in cells treated with metformin and insulin but did not reach statistical significance $(\mathrm{p}=0.06)$ (Fig. 3E).

\section{Discussion}

The intra-abdominal OM adipose tissue has unique anatomic and metabolic characteristics compared to adipose tissues at other locations [36]. Dysfunction of this depot is marked by ectopic fat accumulation and is often associated with hyperinsulinemia and increased risk of T2DM $[37,38]$. Our data report for the first time impairment of the adipogenic capacity of OM preadipocytes taken from obese and morbidly obese IR individuals (including T2DM patients) compared to their IS counterparts, with a potential role of 4-HNE accumulation in this impairment. OM preadipocytes of the IS individuals maintained their capacity to differentiate, potentially allowing superfluous triacylglycerols from the sub-cutaneous depot to be stored within the OM depot, therefore reducing ectopic fat accumulation. This is also supported by better liver function parameters of IS patients reported previously [5]. Our data also show evidence that T2DM patients who were already treated with combined metformin and insulin show improved in vitro adipogenesis compared to those treated with metformin only. This partial rescue of adipogenesis in the combined therapy group was associated with less infiltration of macrophages and 4-HNE accumulation in their OM adipose tissues. Despite no significant differences in circulating TAGs between the two groups, the therapeutic relevance of improved adipogenesis requires verification in a larger cohort. 
A

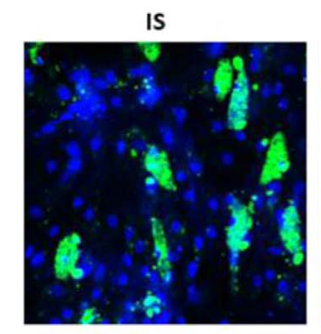

T2DM + Metformin
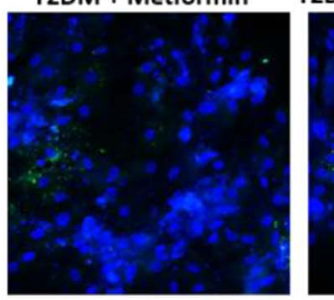

IR

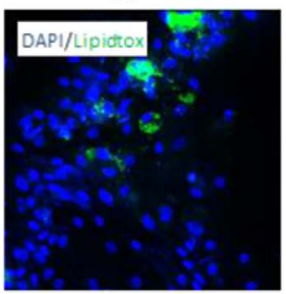

$2 \mathrm{DM}+$ Metformin \& Insulin

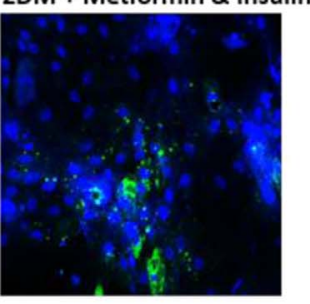

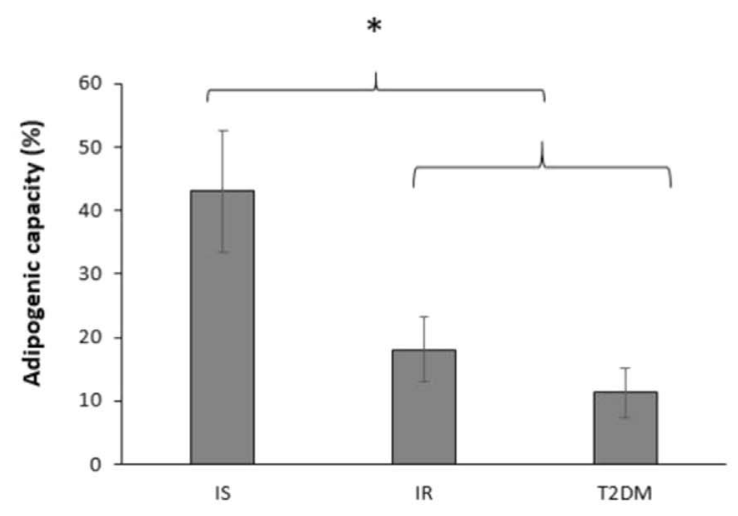

C

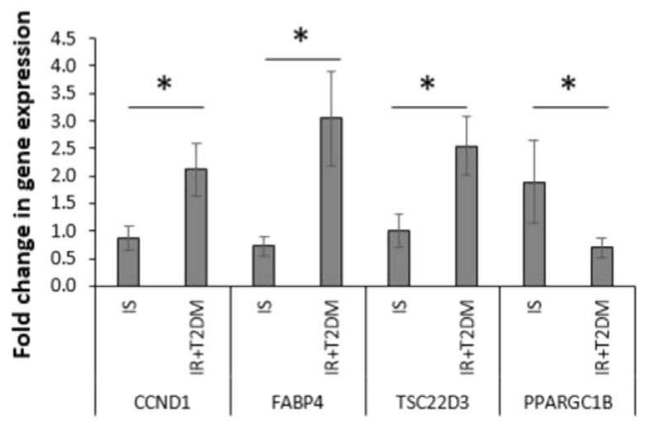

D

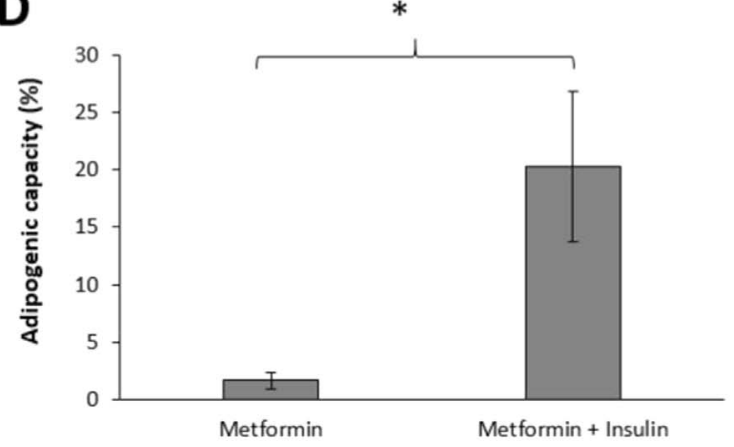

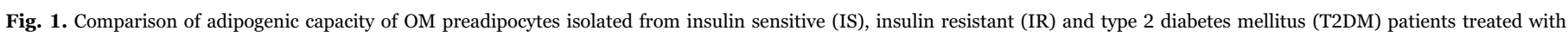

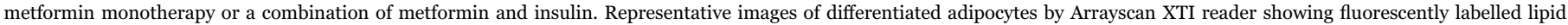

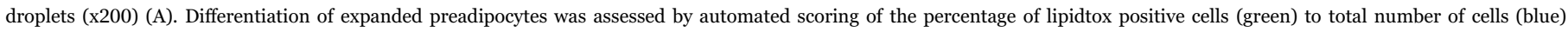

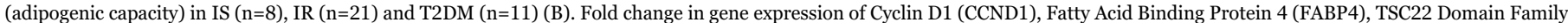

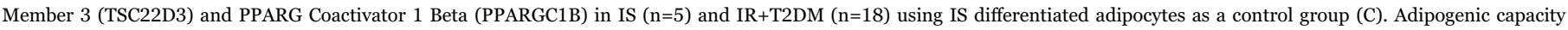

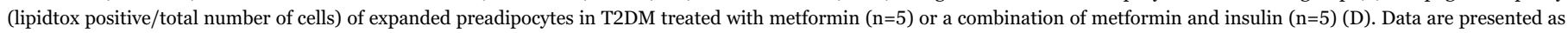
Mean \pm SEM. Differences between groups were tested by ANOVA followed by independent sample $t$-test between IS and all IR (IR+T2DM) groups, ${ }^{*} \mathrm{P}<0.05$.

Insulin resistance-associated impairment of OM adipogenesis was marked by elevated expression of the anti-adipogenic genes CCND1, FABP4 and TSC22D3, all shown previously to reduce PPARG-mediated adipogenesis [39-41], and down-regulation of the pro-adipogenic gene PPARGC1B that lays downstream of PPARG [42]. Impaired adipogenesis was also associated with higher macrophage accumulation and 4HNE staining within the adipose tissues (Fig. 2). Indeed both macrophages and adipocytes are known to promote inflammation and alter cellular redox homeostasis. Mutual interplay between macrophage oxidative burst and ROS derived from adipocyte metabolism of excess nutrients can trigger lipid peroxidation of readily oxidizable adipocyte lipids yielding formation of 4-HNE [9]. As obesity and T2DM are associated with dyslipidemia, 4-HNE may be generated from organs or tissues other than adipose tissues and spread through the blood. This is consistent with the observations in Fig. 2D where high 4-HNE staining was primarily detected in the walls of blood vessels and in the perivascular interstitial tissues. It is likely that 4-HNE is transported while bound to proteins such as albumin since 4-HNE-protein adducts are less easily metabolized than free aldehydes that have high affinity to bind to proteins. In favor of this assumption are also findings of increased levels of 4-HNE-protein adducts in the blood of obese men [15]. Thus, acting as systemic mediator of lipid peroxidation 4-HNE might modulate cell growth, differentiation, apoptosis and paly a role as signaling molecule by its direct action and indirect effect caused by modification of proteins [11]. Moreover, 4-HNE was shown to regulate adipose tissue function and differentiation in both mature and in differentiating adipocytes [13]. Still, the pathophysiological roles of oxidative stress, in particular whether 4-HNE is responsible for insulin resistance, remains to be elucidated [43].

In the current study, T2DM-associated impairment of OM adipogenesis was also associated with increased prevalence of small adipocytes, a finding that was previously attributed to adipocyte hyperplasia associated with greater degree of insulin resistance and T2DM [44]. Possible causative/mechanistic involvement of 4-HNE in such pathophysiology of T2DM-associated impairment of OM adipogenesis is strongly supported by increased levels of 4-HNE observed by immunohistochemical analysis of the OM tissue of these patients (Fig. 2) together with enhanced proliferation and reduced differentiation (adipogenesis) of cultured pre-adipocytes treated with 4-HNE (Fig. 3). This particular product of lipid peroxidation is well known to enhance proliferation and regulate metabolic stress-response and differentiation as it acts as a growth factor for various types of cells [45-48].

Furthermore, the finding of increased ROS production in response to 4-HNE treatment in vitro indicates that 4-HNE might play crucial role in the onset of the vicious etio-pathogenic circle of obesity, 
A

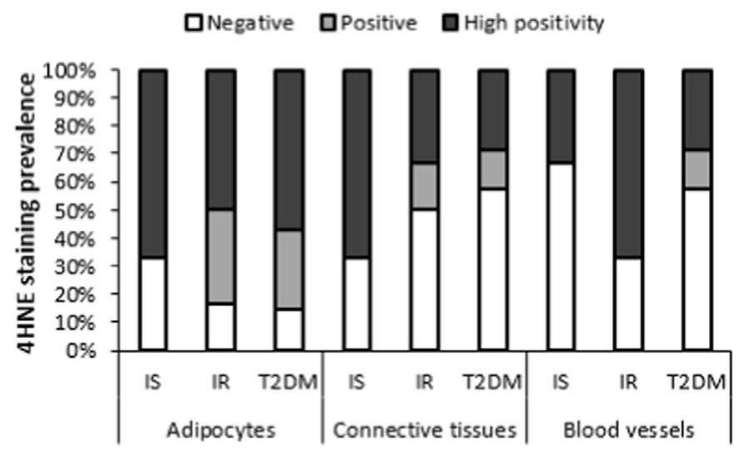

C

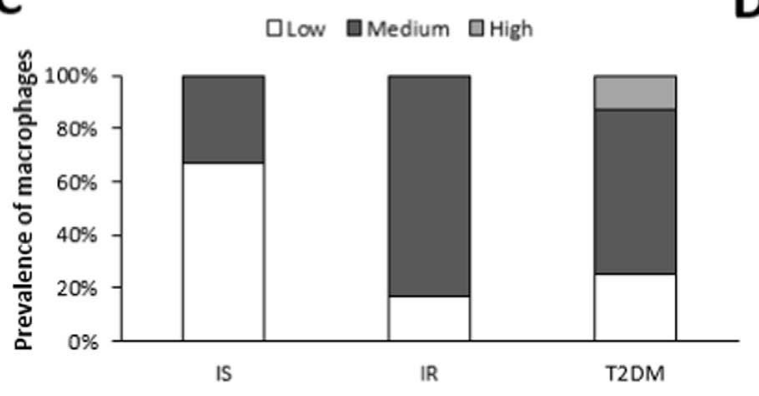

B

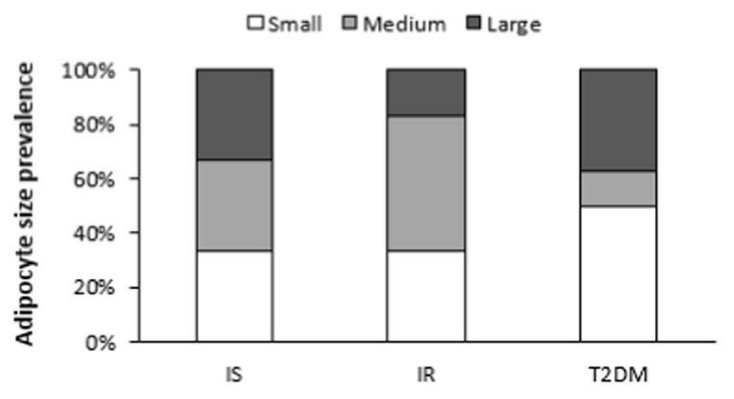

D

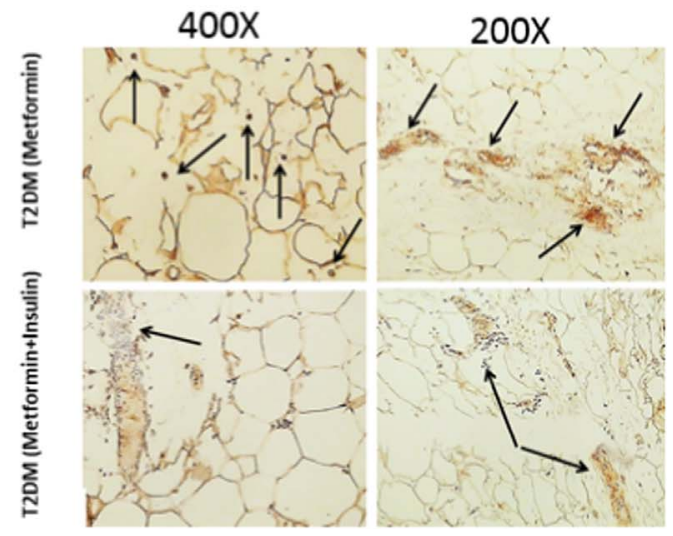

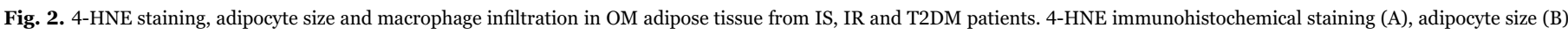

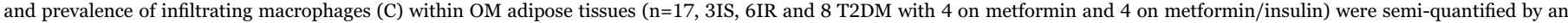

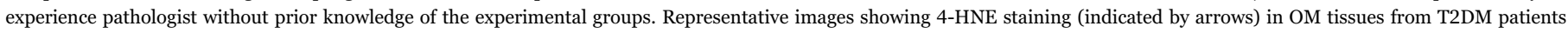
treated with metformin monotherapy (top) or metformin/insulin combined therapy (bottom) (D).

inflammation, oxidative stress and T2DM, which should be further studied to better understand metabolic syndrome and develop improved preventive and therapeutic protocols.

Both metformin and insulin are prescribed therapy protocols for T2DM patients [49]. The synergistic action of insulin with metformin was shown to improve glycemic control over insulin monotherapy [23]. In this study, we hypothesized that the previously reported antiadipogenic effect of metformin could be rescued with co-treatment with insulin. Indeed, the emerging data suggest rescued adipogenesis in the combined therapy group which is marked by lower circulating IL-6 levels and reduced 4-HNE modified proteins concentrations and macrophage infiltration within the adipose tissues. Differences in the adipogenic capacity between the metformin monotherapy and the combination therapy groups may represent a reflection of imprinted memory on the adipose tissue due to exposure to different medications and their various effects on tissue physiology [5]. The superior adipogenesis of combined treatment was further confirmed by treating cells from IR individuals with metformin in the presence of insulin. The 4-HNE treatment reduced adipogenesis, also reported previously $[6,13,21]$, however this phenotype was rescued in the presence of insulin. Furthermore, 4-HNE and metformin significantly induced intracellular adipocyte ROS production in vitro while this effect was also partially blunted by insulin. A similar finding was previously reported in 3T3L1 mouse preadipocytes cell line where treatment with metformin increased ROS production while the combination of metformin and insulin did not [50]. The anti-inflammatory role of insulin [51] in the combined treatment group, manifested by lower circulating IL-6, reduced macrophage infiltration within the adipose tissue and the subsequent lower 4-HNE accumulation and ROS production, may have contributed to improved adipogenesis in these patients although the exact mechanism remains to be investigated. Indeed, the anti-adipogenic roles of IL-6 and 4-HNE in sub-cutaneous tissues from insulin resistance and T2DM were recently suggested [5,6] and the emerging data may suggest a similar role in OM preadipocytes. Fig. 4 represents proposed action of the combined treatment on OM adipogenesis through blunting inflammation and 4-HNE accumulation, as suggested by emerging and recently published data [6].

\section{Conclusions}

Our results demonstrate insulin-resistance associated impairment of $\mathrm{OM}$ adipogenesis and provide a proof of concept that the impaired preadipocyte differentiation and altered cellular redox homeostasis in T2DM patients may be reversible by the synergistic action of insulin and metformin, potentially allowing for a better storage of excess triacylglycerols that otherwise would be deposited ectopically. The involvement of 4-HNE as a potential pathogenic factor and a possible biomarker of impairment of OM adipogenesis in the IR and T2DM patients seems consistent with the recognized role of 4-HNE as a clinical biomarker of different diseases [52,53]. Studies are needed to evaluate these possibilities and explore the underlying pathophysiological mechanisms based on inflammation and oxidative stress.

\section{Funding}

This research was sponsored by Qatar National Research Fund (QNRF), Grant number NPRP6-235-1-048 (MAE, MJ and WK).

\section{Duality of interest}

The authors declare that there is no duality of interest associated with this work. 


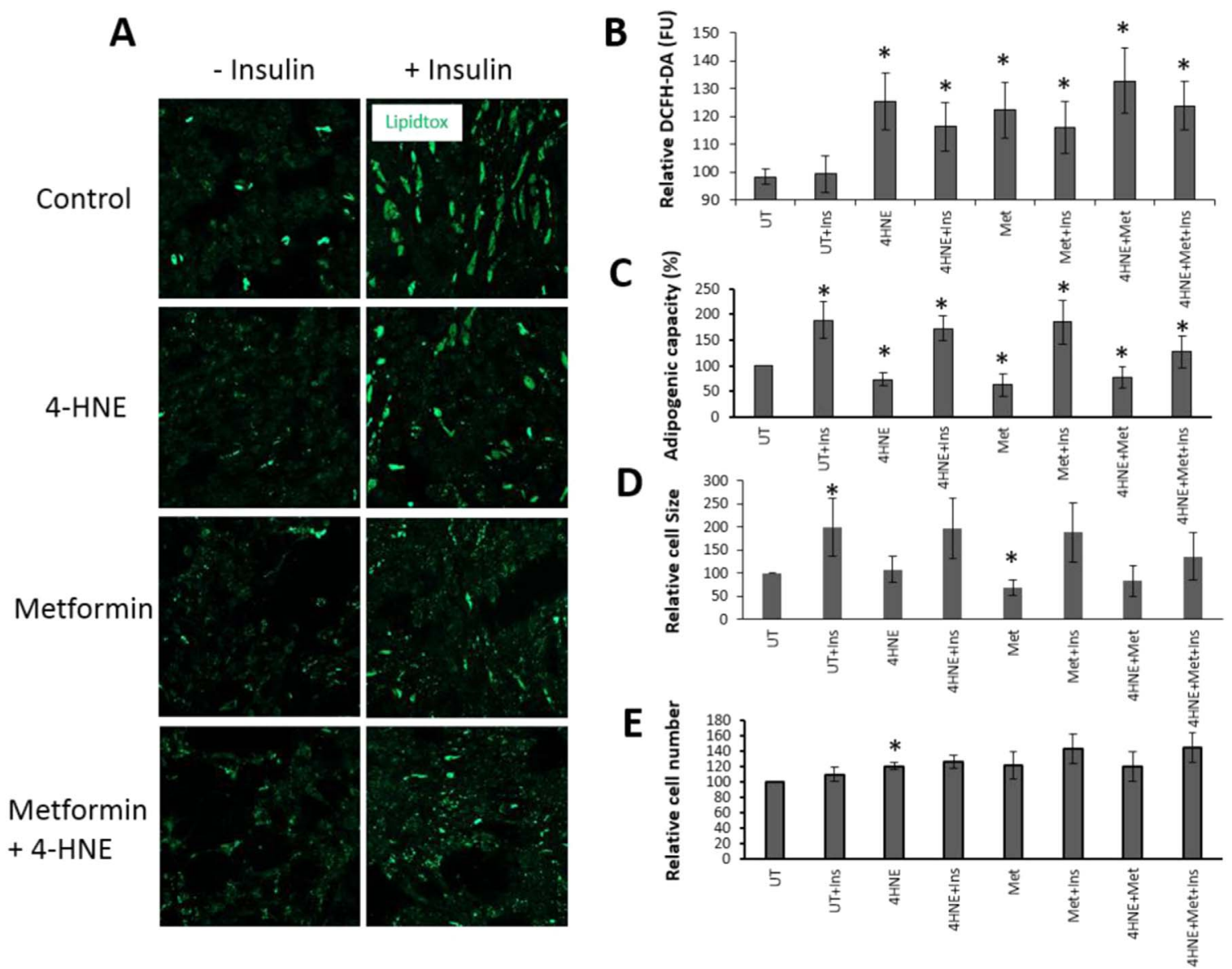

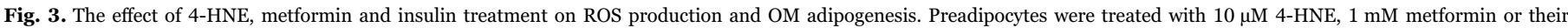

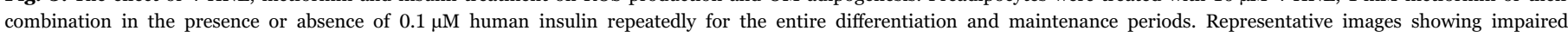

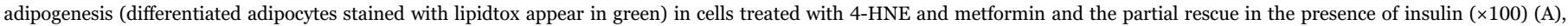

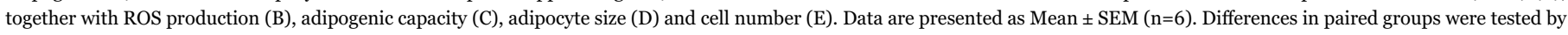
paired samples Test (UT: untreated control, Ins: Insulin, Met: Metformin). P $<0.05$.
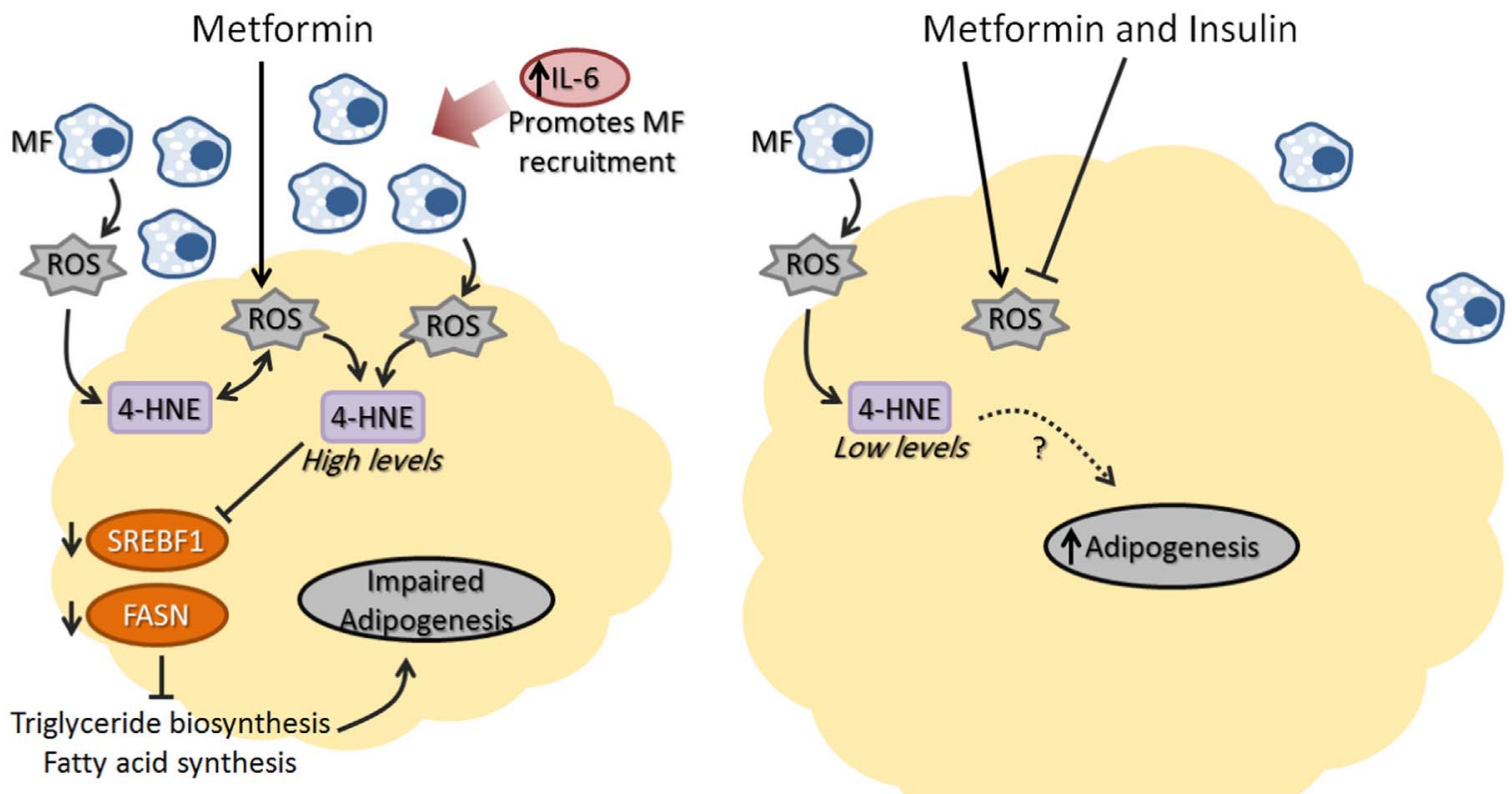

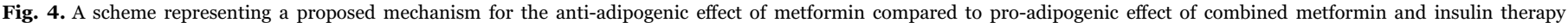
highlighting the roles of oxidative stress (ROS), macrophages (MF) and inflammation. 


\section{Acknowledgments}

We would like to thank Qatar National Research Fund (QNRF) for funding this project (Grant number NPRP6-235-1-048).

\section{Appendix A. Supplementary material}

Supplementary data associated with this article can be found in the online version at http://dx.doi.org/10.1016/j.redox.2017.03.012.

\section{References}

[1] S.E. Kahn, R.L. Hull, K.M. Utzschneider, Mechanisms linking obesity to insulin resistance and type 2 diabetes, Nature 444 (7121) (2006) 840-846. http:// dx.doi.org/10.1038/nature05482.

[2] C. Bogardus, S. Lillioja, D.M. Mott, C. Hollenbeck, G. Reaven, Relationship between degree of obesity and in vivo insulin action in man, Am. J. Physiol. 248 (3 Pt 1) (1985) E286-E291.

[3] J.M. Rutkowski, J.H. Stern, P.E. Scherer, The cell biology of fat expansion, J. Cell Biol. 208 (5) (2015) 501-512. http://dx.doi.org/10.1083/jcb.201409063.

[4] W.P. Cawthorn, E.L. Scheller, O.A. MacDougald, Adipose tissue stem cells meet preadipocyte commitment: going back to the future, J. Lipid Res. 53 (2) (2012) 227-246. http://dx.doi.org/10.1194/jlr.R021089.

[5] S. Almuraikhy, W. Kafienah, M. Bashah, I. Diboun, M. Jaganjac, F. Al-Khelaifi, et al., Interleukin-6 induces impairment in human subcutaneous adipogenesis in obesity-associated insulin resistance, Diabetologia 59 (11) (2016) 2406-2416. http://dx.doi.org/10.1007/s00125-016-4031-3.

[6] M.A. Elrayess, S. Al-Muraikhy, W. Kafienah, A. Al-Menhali, F. Al-Khalaifi, M. Bashah, et al., 4-hydroxynonenal causes impairment of human subcutaneous adipogenesis and induction of adipocyte insulin resistance, Free Radic. Biol. Med. 104 (2017) 129-137. http://dx.doi.org/10.1016/j.freeradbiomed.2017.01.015 (Epub 2017 Jan 12)

[7] S. Furukawa, T. Fujita, M. Shimabukuro, M. Iwaki, Y. Yamada, Y. Nakajima, et al., Increased oxidative stress in obesity and its impact on metabolic syndrome, J. Clin. Investig. 114 (12) (2004) 1752-1761. http://dx.doi.org/10.1172/JCI21625.

[8] A. Higdon, A.R. Diers, J.Y. Oh, A. Landar, V.M. Darley-Usmar, Cell signalling by reactive lipid species: new concepts and molecular mechanisms, Biochem. J. 442 (3) (2012) 453-464. http://dx.doi.org/10.1042/BJ20111752.

[9] M. Jaganjac, O. Tirosh, G. Cohen, S. Sasson, N. Zarkovic, Reactive aldehydes second messengers of free radicals in diabetes mellitus, Free Radic. Res. 47 (Suppl. 1) (2013) S39-S48. http://dx.doi.org/10.3109/10715762.2013.789136.

[10] M. Vazdar, P. Jurkiewicz, M. Hof, P. Jungwirth, L. Cwiklik, Behavior of 4 hydroxynonenal in phospholipid membranes, J. Phys. Chem. B 116 (22) (2012) 6411-6415. http://dx.doi.org/10.1021/jp3044219.

[11] N. Zarkovic, A. Cipak, M. Jaganjac, S. Borovic, K. Zarkovic, Pathophysiological relevance of aldehydic protein modifications, J. Proteom. 92 (2013) 239-247. http://dx.doi.org/10.1016/j.jprot.2013.02.004.

[12] P.A. Grimsrud, M.J. Picklo Sr., T.J. Griffin, D.A. Bernlohr, Carbonylation of adipose proteins in obesity and insulin resistance: identification of adipocyte fatty acidbinding protein as a cellular target of 4-hydroxynonenal, Mol. Cell. Proteom. 6 (4) (2007) 624-637. http://dx.doi.org/10.1074/mcp.M600120-MCP200.

[13] K. Dasuri, P. Ebenezer, S.O. Fernandez-Kim, L. Zhang, Z. Gao, A.J. Bruce-Keller, et al., Role of physiological levels of 4-hydroxynonenal on adipocyte biology: implications for obesity and metabolic syndrome, Free Radic. Res. 47 (1) (2013) 8-19. http://dx.doi.org/10.3109/10715762.2012.733003.

[14] M.P. Mattson, Roles of the lipid peroxidation product 4-hydroxynonenal in obesity, the metabolic syndrome, and associated vascular and neurodegenerative disorders, Exp. Gerontol. 44 (10) (2009) 625-633. http://dx.doi.org/10.1016/j.exger.2009.07.003.

[15] D. Weber, L. Milkovic, S.J. Bennett, H.R. Griffiths, N. Zarkovic, T. Grune, Measurement of HNE-protein adducts in human plasma and serum by ELISAComparison of two primary antibodies, Redox Biol. 1 (2013) 226-233. http:// dx.doi.org/10.1016/j.redox.2013.01.012.

[16] C.J. Bailey, R.C. Turner, Metformin, N. Engl. J. Med. 334 (9) (1996) 574-579. http://dx.doi.org/10.1056/NEJM199602293340906.

[17] D. Kirpichnikov, S.I. McFarlane, J.R. Sowers, Metformin: an update, Ann. Intern Med. 137 (1) (2002) 25-33.

[18] W.C. Knowler, E. Barrett-Connor, S.E. Fowler, R.F. Hamman, J.M. Lachin, E.A. Walker, et al., Reduction in the incidence of type 2 diabetes with lifestyle intervention or metformin, N. Engl. J. Med. 346 (6) (2002) 393-403. http:// dx.doi.org/10.1056/NEJMoa012512.

[19] M. Stumvoll, N. Nurjhan, G. Perriello, G. Dailey, J.E. Gerich, Metabolic effects of metformin in non-insulin-dependent diabetes mellitus, N. Engl. J. Med. 333 (9) (1995) 550-554. http://dx.doi.org/10.1056/NEJM199508313330903.

[20] J.M. Lenhard, S.A. Kliewer, M.A. Paulik, K.D. Plunket, J.M. Lehmann, J.E. Weiel, Effects of troglitazone and metformin on glucose and lipid metabolism: alterations of two distinct molecular pathways, Biochem. Pharmacol. 54 (7) (1997) 801-808.

[21] P. Huypens, E. Quartier, D. Pipeleers, M. Van de Casteele, Metformin reduces adiponectin protein expression and release in 3T3-L1 adipocytes involving activation of AMP activated protein kinase, Eur. J. Pharmacol. 518 (2-3) (2005) 90-95. http://dx.doi.org/10.1016/j.ejphar.2005.06.016.

[22] K.B. Alexandre, A.M. Smit, I.P. Gray, N.J. Crowther, Metformin inhibits intracel- lular lipid accumulation in the murine pre-adipocyte cell line, 3T3-L1, Diabetes Obes. Metab. 10 (8) (2008) 688-690. http://dx.doi.org/10.1111/j.14631326.2008.00890.x

[23] M.G. Wulffele, A. Kooy, P. Lehert, D. Bets, J.C. Ogterop, B. Borger van der Burg, et al., Combination of insulin and metformin in the treatment of type 2 diabetes, Diabetes Care 25 (12) (2002) 2133-2140.

[24] J.A. Scarlett, O.G. Kolterman, T.P. Ciaraldi, M. Kao, J.M. Olefsky, Insulin treatment reverses the postreceptor defect in adipocyte 3-O-methylglucose transport in type II diabetes mellitus, J. Clin. Endocrinol. Metab. 56 (6) (1983) 1195-1201. http:// dx.doi.org/10.1210/jcem-56-6-1195.

[25] R. Goyal, A.F. Faizy, S.S. Siddiqui, M. Singhai, Evaluation of TNF-alpha and IL-6 levels in obese and non-obese diabetics: pre- and postinsulin effects, N. Am. J. Med. Sci. 4 (4) (2012) 180-184. http://dx.doi.org/10.4103/1947-2714.94944.

[26] D.J. Klemm, J.W. Leitner, P. Watson, A. Nesterova, J.E. Reusch, M.L. Goalstone, et al., Insulin-induced adipocyte differentiation. Activation of CREB rescues adipogenesis from the arrest caused by inhibition of prenylation, J. Biol. Chem. 276 (30) (2001) 28430-28435. http://dx.doi.org/10.1074/jbc.M103382200.

[27] H. Sakaue, W. Ogawa, M. Matsumoto, S. Kuroda, M. Takata, T. Sugimoto, et al., Posttranscriptional control of adipocyte differentiation through activation of phosphoinositide 3-kinase, J. Biol. Chem. 273 (44) (1998) 28945-28952.

[28] S. Gesta, Y.H. Tseng, C.R. Kahn, Developmental origin of fat: tracking obesity to its source, Cell 131 (2) (2007) 242-256. http://dx.doi.org/10.1016/ j.cell.2007.10.004.

[29] M. Fischer, K. Timper, T. Radimerski, K. Dembinski, D.M. Frey, H. Zulewski, et al., Metformin induces glucose uptake in human preadipocyte-derived adipocytes from various fat depots, Diabetes, Obes. Metab. 12 (4) (2010) 356-359. http:// dx.doi.org/10.1111/j.1463-1326.2009.01169.x.

[30] S.M. Grundy, H.B. Brewer Jr., J.I. Cleeman, S.C. Smith Jr., C. Lenfant, American Heart A, et al., Definition of metabolic syndrome: report of the National Heart, Lung, and Blood Institute/American Heart Association conference on scientific issues related to definition, Circulation 109 (3) (2004) 433-438. http://dx.doi.org/ 10.1161/01.CIR.0000111245.75752.C6.

[31] B.A. Bunnell, B.T. Estes, F. Guilak, J.M. Gimble, Differentiation of adipose stem cells, Methods Mol. Biol. 456 (2008) 155-171. http://dx.doi.org/10.1007/978-159745-245-8 12.

[32] M.J. Lee, Y. Wu, S.K. Fried, A modified protocol to maximize differentiation of human preadipocytes and improve metabolic phenotypes, Obesity 20 (12) (2012) 2334-2340. http://dx.doi.org/10.1038/oby.2012.116.

[33] M. Poljak-Blazi, M. Jaganjac, I. Sabol, B. Mihaljevic, M. Matovina, M. Grce, Effect of ferric ions on reactive oxygen species formation, cervical cancer cell lines growth and E6/E7 oncogene expression, Toxicol. In Vitro: Int. J. 25 (1) (2011) 160-166 http://dx.doi.org/10.1016/j.tiv.2010.10.013.

[34] M. Jaganjac, M. Poljak-Blazi, R.J. Schaur, K. Zarkovic, S. Borovic, A. Cipak, et al., Elevated neutrophil elastase and acrolein-protein adducts are associated with W256 regression, Clin. Exp. Immunol. 170 (2) (2012) 178-185. http://dx.doi.org/ 10.1111/j.1365-2249.2012.04639.x.

[35] C.M. Spickett, I. Wiswedel, W. Siems, K. Zarkovic, N. Zarkovic, Advances in methods for the determination of biologically relevant lipid peroxidation products, Free Radic. Res. 44 (10) (2010) 1172-1202. http://dx.doi.org/10.3109/ 10715762.2010 .498476

[36] K.N. Frayn, Visceral fat and insulin resistance-causative or correlative?, Br. J. Nutr. 83 (Suppl. 1) (2000) S71-S77.

[37] N. Miyatake, S. Takenami, M. Fujii, Evaluation of visceral adipose accumulation in Japanese women and establishment of a predictive formula, Acta Diabetol. 41 (3) (2004) 113-117.

[38] K.J. Catalano, D. Stefanovski, R.N. Bergman, Critical role of the mesenteric depot versus other intra-abdominal adipose depots in the development of insulin resistance in young rats, Diabetes 59 (6) (2010) 1416-1423. http://dx.doi.org/ $10.2337 / \mathrm{db} 08-0675$.

[39] M. Fu, M. Rao, T. Bouras, C. Wang, K. Wu, X. Zhang, et al., Cyclin D1 inhibits peroxisome proliferator-activated receptor gamma-mediated adipogenesis through histone deacetylase recruitment, J. Biol. Chem. 280 (17) (2005) 16934-16941. http://dx.doi.org/10.1074/jbc.M500403200.

[40] T. Garin-Shkolnik, A. Rudich, G.S. Hotamisligil, M. Rubinstein, FABP4 attenuates PPARgamma and adipogenesis and is inversely correlated with PPARgamma in adipose tissues, Diabetes 63 (3) (2014) 900-911. http://dx.doi.org/10.2337/db130436.

[41] X. Shi, W. Shi, Q. Li, B. Song, M. Wan, S. Bai, et al., A glucocorticoid-induced leucine-zipper protein, GILZ, inhibits adipogenesis of mesenchymal cells, EMBO Rep. 4 (4) (2003) 374-380. http://dx.doi.org/10.1038/sj.embor.embor805.

[42] T. Deng, D.H. Sieglaff, A. Zhang, C.J. Lyon, S.D. Ayers, A. Cvoro, et al., A peroxisome proliferator-activated receptor gamma (PPARgamma)/PPARgamma coactivator 1beta autoregulatory loop in adipocyte mitochondrial function, J. Biol. Chem. 286 (35) (2011) 30723-30731. http://dx.doi.org/10.1074/ jbc.M111.251926.

[43] C.Y. Han, Roles of reactive oxygen species on insulin resistance in adipose tissue, Diabetes Metab. J. 40 (4) (2016) 272-279. http://dx.doi.org/10.4093/ dmj.2016.40.4.272.

[44] L. Fang, F. Guo, L. Zhou, R. Stahl, J. Grams, The cell size and distribution of adipocytes from subcutaneous and visceral fat is associated with type 2 diabetes mellitus in humans, Adipocyte 4 (4) (2015) 273-279. http://dx.doi.org/10.1080/ 21623945.2015.1034920

[45] N. Zarkovic, R.J. Schaur, H. Puhl, M. Jurin, H. Esterbauer, Mutual dependence of growth modifying effects of 4-hydroxynonenal and fetal calf serum in vitro, Free Radic. Biol. Med. 16 (6) (1994) 877-884.

[46] A. Cipak, M. Jaganjac, O. Tehlivets, S.D. Kohlwein, N. Zarkovic, Adaptation to 
oxidative stress induced by polyunsaturated fatty acids in yeast, Biochim. Biophys. Acta 1781 (6-7) (2008) 283-287. http://dx.doi.org/10.1016/j.bbalip.2008.03.010.

[47] S.B. Sunjic, A. Cipak, F. Rabuzin, R. Wildburger, N. Zarkovic, The influence of 4hydroxy-2-nonenal on proliferation, differentiation and apoptosis of human osteosarcoma cells, BioFactors 24 (1-4) (2005) 141-148.

[48] T. Kreuzer, N. Zarkovic, R. Grube, R.J. Schaur, Inhibition of HeLa cell proliferation by 4-hydroxynonenal is associated with enhanced expression of the c-fos oncogene, Cancer Biotherapy Radiopharm. 12 (2) (1997) 131-136. http://dx.doi.org/ 10.1089/cbr.1997.12.131.

[49] B.K. Irons, M.G. Minze, Drug treatment of type 2 diabetes mellitus in patients for whom metformin is contraindicated, Diabetes Metab. Syndr. Obes.: Targets Ther. 7 (2014) 15-24. http://dx.doi.org/10.2147/DMSO.S38753.

[50] A. Anedda, E. Rial, M.M. Gonzalez-Barroso, Metformin induces oxidative stress in white adipocytes and raises uncoupling protein 2 levels, J. Endocrinol. 199 (1) (2008) 33-40. http://dx.doi.org/10.1677/JOE-08-0278.

[51] P. Dandona, A. Chaudhuri, P. Mohanty, H. Ghanim, Anti-inflammatory effects of insulin, Curr. Opin. Clin. Nutr. Metab. Care 10 (4) (2007) 511-517. http:// dx.doi.org/10.1097/MCO.0b013e3281e38774.

[52] W. Luczaj, E. Gindzienska-Sieskiewicz, I. Jarocka-Karpowicz, L. Andrisic, S. Sierakowski, N. Zarkovic, et al., The onset of lipid peroxidation in rheumatoid arthritis: consequences and monitoring, Free Radic. Res. 50 (3) (2016) 304-313. http://dx.doi.org/10.3109/10715762.2015.1112901.

[53] J. Frijhoff, P.G. Winyard, N. Zarkovic, S.S. Davies, R. Stocker, D. Cheng, et al., Clinical relevance of biomarkers of oxidative stress, Antioxid. Redox Signal. 23 (14) (2015) 1144-1170. http://dx.doi.org/10.1089/ars.2015.6317. 